\title{
Beiträge zum Problem des oligarchischen Staatsstreiches in Athen vom Jahre 411.
}

\section{Von Felix Kuberka ${ }^{1}$ ).}

Durch die glanzvollen Untersuchungen Eduard Meyers im zweiten Bande seiner Forschungen zur alten Geschichte über die Revolution der Vierhundert ist das Problem des oligarchischen Staatsstreichs in Athen vom Jahre 411 in ein neues Stadium getreten. Diese Wendung knüpft sich in erster Linie an eine kritischere Bewertung der Akten als diejenige Köhlers und Wilamowitz', welche zuerst das neuerschlossene Material durchforschten und überzengt von der absoluten Zuverlässigkeit des aristotelischen Urkundenberichtes diesen zum Kriterium der thukydideischen Geschichtserzählung erhoben. Wie wenig indes ein solcher Glaube an die unbedingte Zuverlässigkeit aktenmässig bezeugter Tatsachen berechtigt ist, lässt sich aus wenigen methodologischen Bemerkungen ersehen. Gerade in der Durchforschung neuerer Geschichtsperioden sind wir ja sehr oft in der Lage, die aktenmässige Ueberlieferung mit dem anderwärts sicher bezeugten Tatsachenverlauf zu vergleichen, und da zeigt es sich sehr häufig, dass die offizielle, aktenmässige Mitteilung eine von dem realen Geschehen der Dinge völlig abweichende Darstellung gibt. An die von Ed. Meyer angeführten Beispiele, die Ergreifung des Prinzipats durch Augustus und den Stratsstreich rom 18. Brumaire, möge hier nur erinnert werden ${ }^{2}$ ). Das ist ja auch nicht zu verwundern. Sind doch sehr häufig diejenigen, welche die Akten ausstellen, aus diplomatisch-politischen Gründen gar nicht im stande, die volle, unverdrehte Wahrheit voll und ganz herauszusagen, ganz abgesehen nun von den Fällen, wo, wie so oft in revolutionären Zeiten, die nachträglich fixierte Aktenmitteilung lediglich

1) Bei der Redaktion eingegangen am 11. Februar 1907. - Durch die Freundlichkeit der Redaktion habe ich bei der Korrektur meines Aufsatzes noch von Jadeichs Artikel über den Staatsstreich der Vierhundert, Rheinisches Museum Band 62 S. 295 ff. nachträglich Kenntnis erhalten. Eine Aenderung der im folgenden begründeten Auffassung hat mir freilich Judeichs Untersuchung nicht gebracht. Zugunsten der Geschlossenheit meiner Darlegungen möchte ich auf eine nachtrïgliche Polemik gegen Judeichs Ausführungen an dieser Stelle verzichten.

2) Forschungen zur alten Geschichte II, $423 \mathrm{f}$. 
die Aufgabe hat, die dem realen Verlauf der Dinge ermangelnde Legitimität zu geben. Die Möglichkeit einer solchen gleichsam politischen Interpolation ist auch hinsichtlich der aristotelischen Urkundenaufzeichnung wenigstens methodologisch nicht $\mathrm{zu}$ bestreiten. Allein auch wenn wir die Skepsis nicht soweit treiben und den Akten das Vertrauen schenken, das sie verdienen oder nicht, bliebe doch immer noch die Möglichkeit, dass Aristoteles das in den Akten offiziell Mitgeteilte falsch verknüpft und daher die Begebenheiten nicht der Wahrheit entsprechend dargestellt hat. Völlig isoliert und in ihrer urkundlichen Originalität sind ja auch bei Aristoteles die offiziellen Akten nicht erhalten. Diese finden sich vielmehr als Bruchstücke in die historische Darstellung verwoben, die ihrerseits auf mehrere Primärquellen, so auf Thukydides, vielleicht die Atthis des Androtion ${ }^{1}$ ), endlich die uns unbekannte, von der Hand eines Anhängers des Theramenes verfasste oligarchische Parteischrift, für Aristoteles nachweislich auch sonst eine der Hauptquellen, zurïckgeht. Die Möglichkeit, dass bei diesen verschiedenen Grundlagen seines Berichts Aristoteles geirrt und falsche Schlüsse aus den Akten gezogen hat, ist nicht von der Hand zu weisen. Diese methodologischen Erwägungen sind freilich an sich nicht im stande, den aristotelischen Bericht als unzuverlässig und ungenau nachzuweisen. Aber sie zeigen doch wenigstens, wie wenig eine kritiklose Anerkennung des Wahrheitsgehaltes aktenmässig überlieferter Mitteilungen berechtigt ist, und wie sehr wir die Pflicht haben, auch auf die Urkunden die volle Schärfe der historischen Kritik anzuwenden.

Gerade in dieser Beziehung ist aber für die Brüchigkeit des aristotelischen Berichtes von Ed. Meyer der vollgültige Beweis erbracht werden ${ }^{2}$ ). Nur an die Hauptpunkte möge erinnert werden. Wie wir aus dem Eingang cap. 30 sehen, hat nach den entscheidenden Anträgen der Kommission der Dreissig das souveräne Volk tatsächlich zugunsten der Fünftausend abgedankt und auf jede weitere Teilnahme an den Regierungsgeschäften verzichtet. $\mathrm{Da}$ ist es ein schreiender Widerspruch des aristotelischen Berichtes, wenn er die Annahme der in cap. 30, 31 mitgeteilten Verfassungsentwürfe ausdrücklich in eine unter dem Vorsitz des Aristomachos tagende Volksversammlung, $\pi \lambda \tilde{\eta} \vartheta \varsigma_{\varsigma}{ }^{3}$ ) verlegt und völlig unberücksichtigt lässt, dass es eine Vertretung des souveränen Volkes nach dessen Abdankung inmitten des oligarchischen Regimentes der Fünftausend nicht gibt. Auch betreffs der realen Existenz der Fünftausend, ihrer tatsächlichen Konsti-

1) Die Daten cap. 32, 1 sind zweifellos einer Atthis entnommen.

2) Forschungen II, 421, $424 \mathrm{ff}$.

3) Der Versuch Köhlers in Sitzungsberichte der Kgl. Prenssischen Akademie der Wissenschaften 1900 S. 813 das Wort $\pi \lambda \tilde{\eta} \vartheta \circ \varsigma$ im Sinne der Gemeinderersammlung der Fünftausend im Gegensatz zu dem Verfassungsausschuss der Hundert zu fassen (vergl. auch Volquardsen, Verhandlungen der 48. Versammlung deutscher Plitologen und Schulmänner S. 125), ist mit Recht des Sprachgebrauchs wegen abgelehnt worden. 
tuierung und ihrer politischen Anteilnahme zeigt ferner der aristotelische Bericht ein eigentümliches Schwanken. Völlig sicher berichtet ist freilich ihre Einsetzung von Anfang an keineswegs; aber auf ihre faktische Existenz müssen wir doch nach den sonstigen Angaben bei Aristoteles, insbesondere der Nachricht, dass die Fünftausend eine Verfassungskommission von Hundert einsetzen, mit Sicherheit schliessen. In cap. 32, 3 wird auch die Unbegründetheit dieses aus Aristoteles Mitteilungen durchaus bündigen Schlusses dargetan und ausdrücklich den Fünftausend eine bloss nominelle

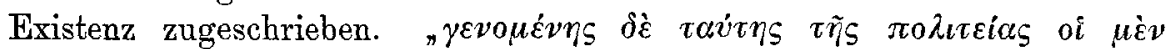

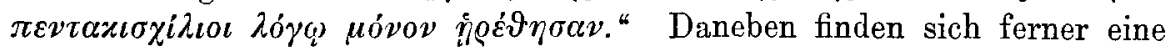
Fülle realer Unmöglichkeiten. Hierher gehört vor allem das Interregnum, das nach der Schilderung cap. 32,1 den Uebergang der radikalen Demokratie (oder gemässigten Oligarchie ?) zu dem revolutionären Regiment der Vierhundert bildet, endlich besonders der formelle Gesamtverlauf der Dinge, die Entwicklung der Bewegung in einer ununterbrochenen Reihe gesetzmässiger Kommissionen und Kommissionsbeschlïsse, in denen sich auf völlig legitimem Wege die Staatsumwälzung im Sinne der Oligarchisch-Radikalen abspielt. Bedenken wir, dass gemäss der aristotelischen Ueberlieferung erst das souverïne Volk zugunsten der Fünftausend abdankt, dann in völlig gesetzmässigen Wahlen erst die 100 , dann die 5000, dann wieder 100 Gesetzgeber, endlich die 400 aufeinanderfolgen, so kann man sich in der Tat mit Ed. Meyer nur darüber wundern, dass in den Rat der Vierhundert doch noch die Oligarchen hineingelangt sind, und warum die antiken Autoren eine solche Bewegung, frei von allen Akten der Gewaltsamkeit und auf friedlichstem Wege vollzogen, überhaupt als Revolution bezeichnen.

Entscheidend ist nun aber, dass, wie zuerst Ed. Meyer mit glücklichem Blick erkannt hat, in der uns unter Lysias' Namen überlieferten Rede für Polystratos ein sicheres Kriterium der damaligen Geschehnisse gegeben ist. Freilich ist gerade in der Verwertung dieses Materials Vorsicht und Skepsis geboten. Denn es unterliegt keinem Zweifel, dass dem juristischen Zweck der Rede entsprechend die Tatsachen zwar nicht erlogen, so doch verdreht und in ein für den Angeklagten möglichst günstiges Licht gerückt worden sind. Indes gewisse Vorgänge, Vorgänge besonders allgemein politischen und verfassungsgemässen Charakters, konnten ihrer allgemeinen Bekanntheit wegen, wenige Monate nach dem Sturz der Vierhundert, doch nicht geleugnet werden, und solche Stellen sind sehr wohl imstande, die Kriterien für die Richtigkeit des uns überlieferten Materials abzugeben.

Es ist bekannt, dass gegenüber der lebenswollen Schilderung des Thukydides sich der Bericht des Aristoteles als dürftige Aktenmitteilung ausnimmt. Die Vorgeschichte der Revolution, die Ereignisse in Samos und Athen übergeht er vollständig; erst wo die Akten zu uns reden, setzt 
eine etwas breitere Darstellung ein. Nachdem Melobios eine einleitende Rede gehalten hat, erfolgt als erster Antrag der Antrag des Pythodoros von Anaphlystos dahingehend, zu den vorhandenen Probulen noch 20 andere über vierzig Jahre alte Männer zu wählen mit der Bestimmung,

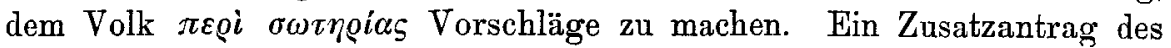
Kleitophon besagt, bei der Aufstellung der Vorschläge auch die Gesetze des Kleisthenes hinzuzuziehen - ein Antrag, der offenbar auf die gemässigte Reformpartei zurückgeht. Fehlt dieser Zusatzantrag auch als unwesentlich in den Angaben der thukydideischen Erzählung, so wird uns doch alles übrige vollkommen gemäss des Antrags des Pythodoros von Anaphlystos berichtet. Nur gibt Thukydides die Anzahl der Mitglieder

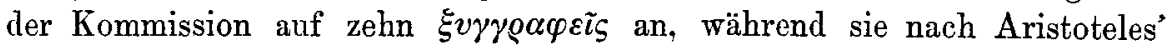
Bericht sich mit Einschluss der Probulen auf dreissig beliefen. Durch die bei Harpokration und Suidas überlieferten Angaben, die im wesentlichen die Richtigkeit der aristotelischen Darstellung erweisen, ist Thukydides in diesem Falle eines nicht unwesentlichen Irrtums überwiesen worden 1). Um so mehr zeigen sich im übrigen die beiden Quellendarstellungen gleichmässig und übereinstimmend unterrichtet. Dass es sich bei Thuky-

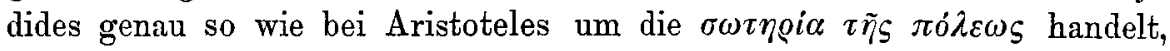
ist genau so klar aus der historischen Erzählung des Thukydides wie der offiziellen Aktenmitteilung des Aristoteles ersichtlich. Nur unter der Vorspieglung, dass es bei der gegenwärtigen Verfassungsänderung den

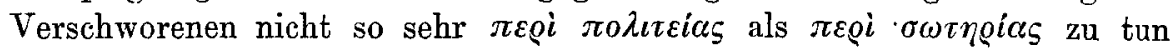
ist, bringen die Oligarchen die Einwilligung des Demos in die Verfassungsänderung zustande (cap. 53,3). Mehr dagegen bietet Thukydides den bestimmten Termin, die $\dot{\eta} \mu \dot{\varepsilon} \varrho \alpha$ $\oint \eta \tau \dot{\eta}$ sowie die Charakterisierung der

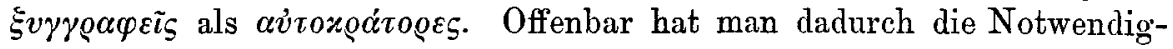
keit der Begutachtung der Kommissionsanträge durch den alten Rat umgehen wollen, dessen man in seiner Majorität doch nicht sicher war ${ }^{2}$ ). Das beweist zugleich, dass selbst in den konservativeren Kreisen die revolutionär-oligarchische Bewegung doch nur zum Teil durchgedrungen war.

Es folgen, da Thukydides den von Aristoteles namhaft gemachten Zusatzantrag des Kleitophon nicht erwähnt, die entscheidenden Anträge auf dem Kolonos. Dass zunächst zwei Anträge eingebracht werden, der eine zur Geschäftsordnung von vorbereitender Bedeutung, der zweite die positiven Vorschläge der Verfassungsänderung enthaltend, wird von Aristoteles und Thukydides übereinstimmend berichtet. Auch ist kein $\mathrm{Z}_{\text {weifel, }}$

1) Ed. Meyer, Forschungen II, 417. Köhler, Sitzungsberichte 1900 S. 807 . Busolt, Griechische Geschichte III, 2 S. 1477 Anm. Einen Ausweg versucht Volquardsen, Verhdl.

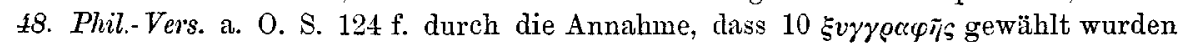
nebst zehn $\pi \dot{x} \varrho \varepsilon \delta \varrho o \iota$ und mit der Verpflichtung, die zehn $\pi \rho \dot{\beta} \beta o v \lambda o \iota$ hinzuzuziehen. Worauf sich diese Annahme stützt, ist leider nicht ersichtlich.

2) Köhler, Sitzungsberichte 1900 S. 807 Anm. 
dass inhaltlich der erste Antrag des Aristoteles betreffs der völligen Freiheit der politischen Rede und der Strafbarkeit aller Zuwiderhandelnden mit den Angaben bei Thukydides übereinstimmt. Nur gibt Aristoleles den vollen Wortlaut des Psephisma wieder, während Thukydides denselben in seiner Weise in einen kurzen historischen Bericht umsetzt: $\dot{\varepsilon} \xi \varepsilon i v a \iota$

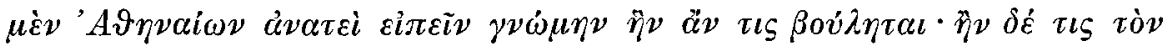

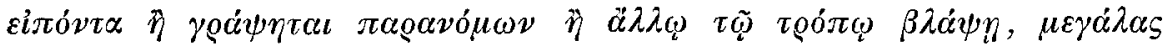

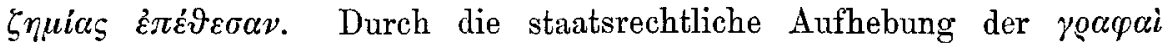
$\pi \alpha \varrho \alpha \nu \mu^{\mu} \omega \nu$ soll offenbar die persönliche Sicherheit der Oligarchen gewährleistet werden. Es entspricht daher nur dem natürlichen, logischen

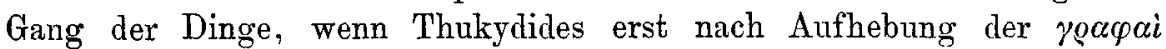
$\pi a \alpha_{\nu} \mu_{\mu} \omega \nu$ die Oligarchen mit ihren positiven, auf Umsturz der Verfassung hinzielenden Anträgen hervortreten lässt.

Allein hinsichtlich dieser materiellen Anträge gehen nun Thukydides und Aristoteles beträchtlich auseinander. Schon in der äusseren Antragstellung ist eine wesentliche Differenz zwischen Aristoteles und Thukydides vorhanden. Nach Aristoteles' Darstellung ist es kein Zweifel, dass auch die positiven materiellen Anträge Anträge der Kommission der Dreissig sind. Nach Thukydides ist ihr eigentlicher Urheber und Antragsteller Peisander. Mehr noch dagegen als in dieser formalen scheinen die Anträge bei Thukydides und Aristoteles in materialer Hinsicht abzuweichen. Zwar die Abschaffung der Besoldung wird von Aristoteles und Thukydides übereinstimmend berichtet. Aber schon die von Thukydides im Eingang des Antrags namhaft gemachte Reorganisation der Aemter geht beträchtlich über die von Aristoteles gemachten Mitteilungen hinaus. Völlig unvergleichbar mit Aristoteles sind aber die folgenden Vorschläge: die Wahl der 5 Proedroi, die durch diese zu erfolgende Ernennung der Hundert, die Ergänzung der Hundert durch Kooptation zu einem souverän die Staatsgeschäfte führenden und nach Gutdünken die 5000 auswählenden Rat von 400, während Aristoteles nur die Beschränkung der politischen Bürgerschaft auf nicht weniger als 5000 und die Wahl derselben durch 100 von den Phylen gewählte, über vierzig Jahre alte Männer erwähnt. Von diesen kontrastierenden Bestimmungen ist höchstens die $100 \mathrm{Koop}-$ tationskommission bei Thukydides mit Aristoteles Wahlkommission von 100 vergleichbar. Allein abgesehen von ihrer zufälligen Mitgliederzahl sind doch nach ihren Bestimmungen und verfassungsgemässen Aufgaben die Kommissionen grundverschieden. Nach Aristoteles erhält die Kommission nur die Aufgabe, die 5000 nach feierlichem Eidschwur auszuwählen. Nach Thukydides wählen die 100 nicht die 5000, sondern die 400 und bestehen, mit allen Rechten der Souveränität ausgestattet, als Kern und fester Grundbestandteil des neuen oligarchischen Rates fort. Kein Zweifel, dass vor allem sie den Umsturz der Dinge im Jahre 411 betrieben baben. Von einer solchen revolutionären und politisch ausserordentlich bedeutsamen 
Tätigkeit der Hundert ist in dem offiziellen Aktenbericht des Aristoteles nicht die Rede ${ }^{1}$ ). Wie ferner Busolt ${ }^{2}$ ) sehr richtig bemerkt hat, setzt freilich auch der bei Thukydides den 400 gegebene Auftrag, die 5000 auszuwählen, einen Beschluss über die Einsetzung von 5000 voraus, und in der Tat wird uns von Thukydides selbst in dem Programm der Oligarchen cap. 65, 3 von einer Uebertragung der Staatsgewalt auf nicht mehr als 5000 berichtet. Allein sehr befremdlich wird von Thukydides dieser Antrag in seiner offiziellen Fassung selbst nicht erwähnt, überdies in dem Programm der Oligarchen die Zahl 5000 nicht wie in Aristoteles Antrag als die Minimal-, sondern die Maximalzahl angegeben. Offenbar hätte diese wesentliche Differenz von Thukydides berichtet werden müssen ${ }^{3}$ ). So ist sowohl in formaler wie materialer Beziehung zwischen Thukydides und Aristoteles nur eine grosse Differenz zu konstatieren, und die Frage nach der Wahrheitsgemässheit der kontrastierenden Darstellungen würde ungelöst bleiben, wenn nun nicht neben den beiden Quellen, dem thukydideischen Bericht und der aristotelischen Ueberlieferung, uns noch eine dritte in die Ereignisse der Bewegung von 411 unmittelbar einführende Quelle als objektiver Massstab der Geschehnisse gegeben wäre. In dieser Verwertung als materiales Kriterium liegt für unser Thema die entscheidende Bedeutung der Rede für Polystratos.

Wir erfahren aus derselben folgendes:

1) Beschlossen ist, Fünftausend die Staatsgewalt zu übergeben. Von der Verteidigung wird dieser Beschluss indirelit mehrmals hervorgehoben.

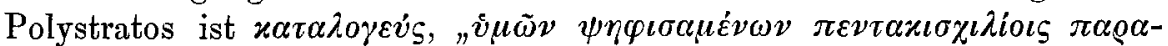

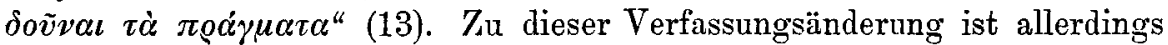
das Volk und so auch Polystratos von den Machthabern unter den späteren Vierhundert verleitet worden (16). Ein Vorwurf ist darum aus der Verfassungsïnderung Polystratos ebensowenig wie den übrigen Anhängern der Demokratie zu machen.

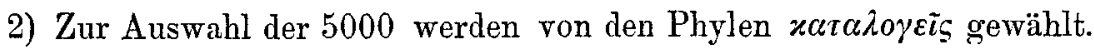
Bevor sie ihr Amt antreten, haben sie einen Eid zu leisten - offenbar gewissenhaft die Auswahl der 5000 durchzuführen. Dass Polystratos von den Phylen gewählt worden ist, lässt am besten die Echtheit seiner de-

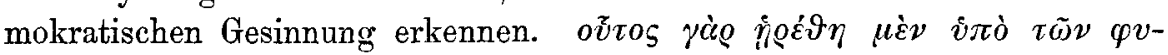

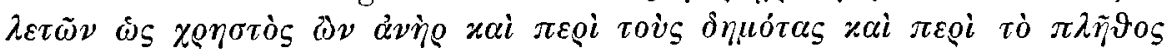

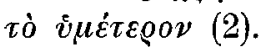

1) Aehnlich Volquardsen a. a. O. S. 126: Die Hundertkommissionen bei Thukydides und Aristoteles sind verschieden a) nach dem Ursprung (Kooptation gegenüber Wahl aus den Phylen), b) nach dem Zweck (Bildung des Rates der Vierhundert gegenüber der Konstituierung der Fünftausend), c) nach dem Lebensalter ihrer Mitglieder (dreissig Jahr gegenüber vierzig Jahren).

2) Busolt, Griechische Geschichte III, 2 S. 1480 Anm.

3) Mit Busolt gegen Ed. Meyer, Forschungen II, 419 f. 


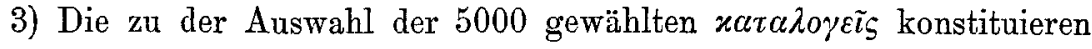
sich zu einem die Regierungsgeschäfte autonom führenden Kat. Daraus, dass Polystratos sofort nach Eidesschwur in den Rat eintritt, ist zu folgern, dass die Ablegung des Eides tatsächlich den offiziellen Eintritt in den Rat in sich schloss ${ }^{1}$ ). Uebrigens hat Polystratos nur gezwungen an

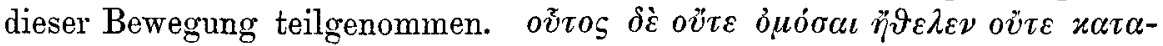

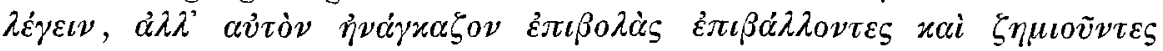
(14). Daher ist er auch, nachdem er 8 Tage in dem Rat gesessen hat, als Kommandant nach Eretria abgefahren.

Vergleichen wir diese sicher feststehenden Tatsachen mit den in Aristoteles' Anträgen vorliegenden Bestimmungen, so ist zunächst die völlige Uebereinstimmung des Punktes 1 und 2 mit Aristoteles' materiellem Antrag der Kommission der Dreissig hervorzuheben. Die Uebertragung der Staatsgewalt auf 5000, die Bestellung einer Wahlkommission für die 5000, die vor dem Wahlgeschäft einen bestimmten Eid ablegt, sowie deren Wahl durch die Phylen - alles dies wird uns durch die Rede für Polystratos in völlig einwandfreier Weise bestätigt. Nur lässt uns die Verteidigung - höchst wahrscheinlich aus sehr bewussten Gründen - über die Anzahl der $x \alpha \tau \alpha \lambda o \gamma \varepsilon \tilde{l}_{S}$ absichtlich im Unlilaren. Da indes in allen übrigen Momenten, dem Zweck ihrer Einsetzung, der Verpflichtung zum Eide und der Altersbestimmung - Polystratos ist siebzigjährig - die Behörde der

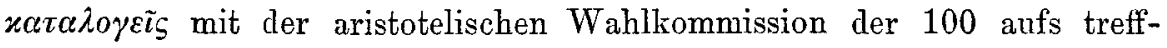
lichste übereinstimmt, dürfen wir mit völliger Sicherheit die Zahl 100 von der aristotelischen Wahlkommission auch auf die in Frage stehende Be-

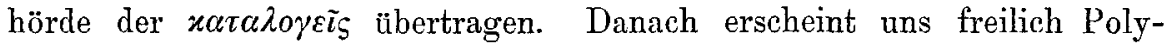
stratos' Verhalten in einem viel ungünstigeren Lichte. Er hat zweifellos nicht nur im allgemeinen zu den Anhängern der Oligarchie gelört, sondern auch in dem eigentlichen Kern der Machthaber der 400 Sitz und Stimme besessen und darum ohne Frage auch in der Entwicklung der revolutionären Bewegung eine aktiv hervorragende Stellung eingenommen.

Ebendarin gehen nun Aristoteles und die Rede für Polystratos weit auseinander. Nach Aristoteles' Darstellung ist kein Zweifel, dass zunächst dem Kommissionsantrag der Dreissig entsprechend die Wabl der 5000 durch die von den Phylen gewählte Kommission von 100 vor sich geht

1) Etwas zu weit geht Wilamowitz mit der Behauptung, dass, die Wahl zum

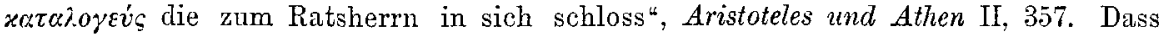
aber umgekehrt die $x \alpha \tau \alpha \lambda .0 \gamma \varepsilon \tilde{c} \zeta$ eo ipso zu Mitgliedern des Rates wurden, was Volquardsen a. a. $0 . \$$. 126 bestreitet, ergibt sich $\$ 14$ aus der unmittelbaren Folge von

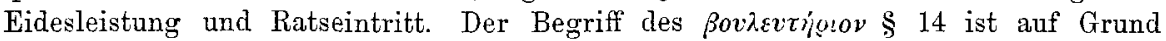
einer Vergleichung der $\$ \$ 1,7,14$ und 16 in der Tat im Sinne der Ratsversammlung,

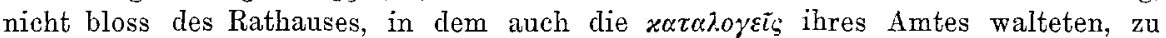
verstehen. Aus der subjektiven, übrigens sehr unlogischen Motivierung des Verhaltens des Angeklagten von seiten der Verteidigung $\$ \$ 3 \mathrm{ff}$., 14 ist ein Einwand gegen diese Interpretation nicht herzuleiten (gegen Volquardsen a. a. O. S. 127). 
und demgemäss die 5000 in der Tat als die neue souveräne Körperschaft verwirklicht werden. Nach Polystratos' Verhalten wählen die Hundert nicht die 5000, sondern konstituieren sich sofort nach abgelegtem Amtseid offenbar, da ihre ursprüngliche Zahl 100 beträgt, durch Kooptation - zu einem Rat von 400 , der nun seinerseits den alten Rat der 500 ablöst und statt der 5000 mit souveräner Machtvollkommenheit die überkommene Staatsgewalt ausübt. In dem Rat der 400 sind also zwei Kategorien: 100 von den Phylen Gewählte und 300 von den Hundert durch Kooptation Ernannte ${ }^{1}$ ). Von allen diesen durchaus revolutionären und die früheren Anträge anullierenden Begebenheiten ist in dem offiziellen Aktenbericht des Aristoteles nicht die Rede. Diese Tatsache lässt nur auf eine Unvollständigkeit der von Aristoteles mitgeteilten Akten schliessen. Was die

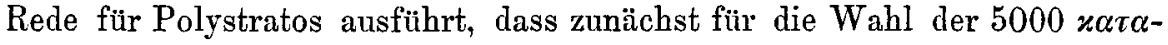
2oyeĩs phylenweis gewählt werden, dass sich aber dann die Behörde der

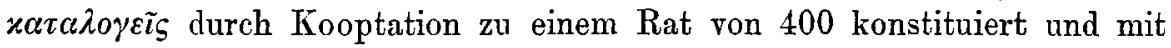
souveräner Machtbefugnis die Staatsgewalt ausübt, musste in gewissen, zweifellos offiziellen Anträgen damals zum Vorschlag und Durchführung gebracht werden. Mag Aristoteles diesen Antrag, was freilich sehr unwahrscheinlich ist, eigenmächtig fortgelassen, oder aber, was viel wahrscheinlicher ist, nicht gekannt haben ${ }^{2}$ ), Tatsache ist, dass über die Umwandlung der $\varkappa \alpha \tau \alpha \lambda o \gamma \varepsilon \tilde{l}_{S}$ in den Rat der 400 gemäss Polystratos' Verhalten tatsächlich beschlossen sein muss, und dass in den Akten, so wie sie uns bei Aristoteles vorliegen, zweifellos Anträge entscheidendster Bedeutung fehlen.

Ich behaupte nun, dass dieser bei Aristoteles vermisste, aber aus Polystratos' Verhalten unmittelbar zu erschliessende Antrag in voller Offenheit in dem historischen Bericht des Thukydides vorliegt. Stellen wir uns die Entwicklung bis zu dem bisher erreichten Punkt des materiellen Antrags der Kommission der Dreissig noch einmal klar vor Augen. Nach den formellen und materiellen Anträgen der Kommission der Dreissig ist

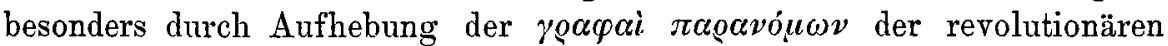
Bewegung Tür und Tor geöffnet und demgemäss im Sinne der gemässigten Oligarchen die Uebertragung der Staatsgewalt auf 5000 beschlossen worden. Allein in diesem Regiment eines Rates von 5000 sahen, wie Thukydides berichtet, die Radikalen der Oligarchen geradezu Demokratie (cap. 92,11), und zweifellos war es so nicht ihr Bestreben, sich mit diesem im Sinne der Extremen allzu liberalen Antrag zu begnügen. Wer aber hätte die Bewegung besser und glücklicher weiterführen können als der Mann, der schon von Anfang an als der eigentliche Macher inmitten der revolutionär-oligarchischen Bewegung gestanden und alles bisher zum glück-

1) Meyer, Forschungen II, 428.

2) Beides Vermutungen Ed. Meyers, Forschungen II, $429 \mathrm{f}$. 
lichsten Ergebnis geführt hatte: ich meine Peisander? Ein Antrag des Peisander liegt uns nun aber bei Thukydides vor, von ihm persönlich eingebracht und zu den Anträgen bei Aristoteles sich in untilgbarem Widerspruch befindend. Alles, was wir bei Aristoteles der Rede für Polystratos gemäss vermissen, finden wir in diesem Antrag des Peisander wieder. Es wird beantragt, die Aemter umzugestalten und die Besoldung aufzuheben, fünf Proedroi zu wählen, diese sollen 100 wählen und diese sich zu einem Rat von 400 ergänzen, der mit souveräner Machtrollkommenheit die Staatsgewalt ausüht und, wenn es ihm beliebt, die 5000 auswählt. Es unterliegt daher gar keinem Zweifel, dass nach dem materiellen Antrag der Kommission der Dreissig von Peisander noch persönlich ein Zusatzantrag eingebracht ist, inhaltlich die Ueberleitung der oligarchischen Wahlkommission der Hundert in den Rat der 400 in sich schliessend und geschichtlich das entscheidende Moment der revolutionär-oligarchischen Bewegung enthaltend. Nachdem im Sinne der Gemässigten der Beschluss auf Einsetzung eines Rates von 5000, wie ihn Aristoteles berichtet und Thukydides' Antrag in der 'Tat roraussetzt, durchgegangen ist, wird durch den späteren Zusatzantrag des Peisander jener frühere Antrag zugleich formell mit gewissen Ergänzungsbestimmungen umkleidet, tatsächlich und historisch aber dem Wunsch der Radikalen entsprechend zugleich mit dieser umgestaltenden Fassung anulliert. Mochte man formell immerhin noch an der Konstituierung der 5000 als dem leitenden Endziel der Entwicklung festhalten, tatsächlich lag in der Konstituierung des Rates der 400 das eigentliche Endziel der oligarchischen Bewegung und war mit dem Antrag des Peisander der Abschluss der oligarchischen Umwälzung, soweit diese sich wenigstens auf scheinbar legitimem Wege abspielte, erreicht. Mit der Ernennung der 5000 mochte sich dann die ferne, unbegrenzte Zukunft, je nachdem die Dinge im Sinne der radikalen Oligarchen günstig oder ungünstig lagen, abfinden. Dieser Auffassung könnte nur die Tatsache entgegengehalten werden, dass, wie Thulidides berichtet, die Volks-

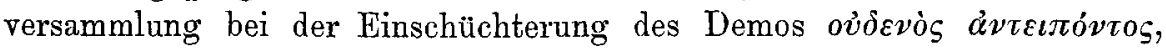
ohne Rede und Gegenrede geschlossen und aufgelöst worden ist (cap. 69,1). Allein zu einer wenn auch nur scheinbaren Debatte ist es zweifellos doch - schon zur Täuschung der Nichtverschworenen - gekommen. Und gerade Thukydides berichtet ja selber, dass weder im Rat noch in der Volksversammlung jemand den Verschworenen zu widersprechen wagte, dass indes die Redner in der Ekklesie und dem Rat auch der Partei dieser angehörten und alles, was sie vorbrachten, schon vorher abgekartetes Spiel war (cap. 66). Aehnlich hat sich offenbar auch in der Volksversammlung auf dem Kolonos der Scheinakt alter Volkssouveränität zugetragen. Die Rede für Polystratos ist zugleich der sicherste Beweis dafür, dass trotz des Antrags der 30-Kommission der Zusatzantrag des Peisander, was ja auch bei dem Terrorismus der Machthaber sehr begreiflich, ungehindert durchgegangen ist. Nur die Wahl 
der 100 durch die in Peisanders Antrag vorgesehenen fünf Proedroi ist zweifellos nicht historische Wirklichkeit geworden. Denn dass die 100 tatsächlich von den Phylen, nicht von den Proedroi ausgewählt wurden, steht durch die Angaben der Rede für Polystratos fest. Es ist relativ gleichgültig, wie wir uns diesen Sachverhalt erklären mögen. Möglich ist, dass jene Bestimmung nur ursprünglich in dem Antrag des Peisander stand, dass sie aber nachträglich in dem entscheidenden Moment in dem vorgetragenen Antrag ihres allzu reaktionären Aussehens halber - konnte man doch auch ohne sie völlig zum Ziel gelangen - weggelassen wurde. In Thukydides' Bericht hätte sich so noch ein rudimentäres Gliéd des ursprünglichen schriftlichen Antrags Peisanders, der Thukydides allein als Quellennachricht vorlag, erhalten. Wesentliches wird aber durch die ganze Proedroifrage an der Entwicklung nicht berührt.

Durch die obigen Ausführungen wird zugleich das Verhältnis des aristotelischen und thukydideischen Berichtes in wesentlichen Stücken zu einander abgeändert. Immer glaubte man bisher, in dem Antrag bei Aristoteles und dem bei Thukydides vorliegenden Antrag des Peisander ein und denselben historischen Antrag nur in seinen verschiedenen Fassungen erblicken zu mïssen. Daher bestand alles Bestreben darin, jene beiden Anträge miteinander zu vergleichen, durch die Vergleichung selbst die Widersprüche festzustellen und, wenn möglich, diese teils auf ein Minimum zurïckzuführen, teils zu versöhnen und zu mildern. Jetzt zeigt es sich, dass diese Anträge in ihren Bestimmunıren wie verfassungsgemässen Festsetzungen überhaupt nicht vergleichbar, dass sie zeitlich unterschieden und de facto völlig verschiedenen Ursprungs sind. Ist der Antrag des Peisander, wie ibn Thukydides mitteilt, erst später nach den Kommissionsanträgen als spezieller Zusatzantrag eingebracht worden, dann ist zwar wohl zwischen dem thukydideischen und aristotelischen Bericht ein logischer Widerspruch, der sich aus den widersprechenden Anträgen selbst herschreibt, zu konstatieren, allein eine wirkliche historische und tatsächliche Differenz nicht mehr zu behaupten und an die Stelle des ausschliessenden Verhältnisses des Entweder - oder das ergänzende Verhältnis des Sowohl - als auch gesetzt. Aristoteles berichtet völlig richtig; wenn er auf den von der Kommission der Dreissig vorgelegten formellen Antrag den materiellen Antrag derselben folgen lïsst und zunächst über die Wahl eines Rates "von 5000 durch eine Wahlkommission von 100 berichtet. Aber Aristoteles kennt nicht den historisch allein entscheidenden Zusatzantrag des Peisander und daraus folgt, dass von ihm der reale Verlauf der Dinge völlig irrtïmlich, obwohl anscheinend völlig in Uebereinstimmung mit den freilich unvollständigen Akten dargestellt wird. Wiederum haben sich bei Thukydides sowohl der formelle Antrag der Kommission wie der persönliche Zusatzantrag des Peisander erhalten. Nur ist ihm wieder der materielle Antrag der Kommission - die Fassung des Programms cap. 65, 3 
braucht, wie Busolt ${ }^{1}$ ) richtig bemerkt, keineswegs aus einer direkten Kenntnis des bei Aristoteles erhaltenen Antrags berzurühren ${ }^{2}$ ) - fremd geblieben, und zweifellos irrt darum Thukydides, wenn er die Kommission

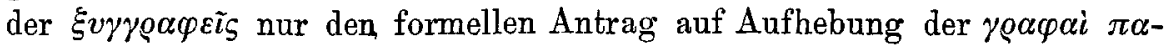

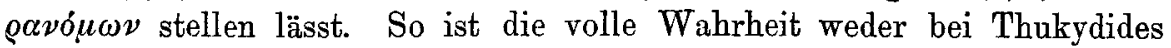
noch bei Aristoteles vorhanden. Aber während Aristoteles nur die offiziellen Anträge der Kommission der Dreissig kennt, ist von Thukydides doch zugleich der historisch allein entscheidende Zusatzantrag Peisanders verwertet worden, und das hat zur Folge, dass jede Darstellung der geschichtlichen Bewegung der Vierhundert in erster Linie die sachlich allein wahrheitsgemässe und mit dem Geist und der Lebendigkeit des grossen Historikers geschriebene Ueberliefernng des Thukydides zugrunde legen muss ${ }^{3}$ ).

Auf einige Bemerkungen im Detail möge noch eingegangen werden. Es erledigt sich zunächst die Frage, ob entsprechend der Darstellung des Aristoteles die Kommission der Dreissig oder aber, wie Thukydides berichtet, tatsächlich Peisander der formelle Antragsteller der materiellen Verfassungsvorschläge gewesen ist. Einen Widerspruch hat freilich Ed. Meyer auch unter dem Gesichtspunkt einer Identität des Antrags bei Thukydides und Aristoteles in diesen Differenzen nicht erblicken wollen. „Ein-

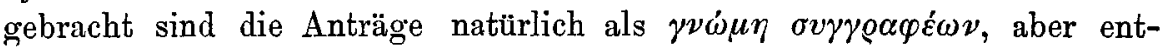
worfen und vermutlich auch vorgetragen hat sie Peisander, der ja zweifellos unter ihnen gewesen ist ${ }^{4}$ ). Indes sind damit, wie Busolt ${ }^{5}$ ) und Köhler ${ }^{6}$ ) mit Recht betonen, die Schwierigkeiten nicht beseitigt. Ausdrücklich unterscheidet ja Thukydides die Anträge nach den Antragstellern: jener erste ist der Antrag der Kommission, dieser, während die Kommission nach Auf-

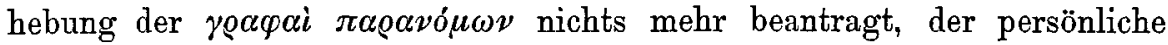
Antrag des Peisander. Es ist nach dem bisher Ausgeführten kein Zweifel, dass von einer Differenz zwischen Thukydides und Aristoteles in diesem Punkte nicht die Rede sein kann, aber nicht in dem Sinne Eduard Meyers,

1) Griechische Geschichte III, 21480 Anm.

2) Dass Thukydides auch den formellen Antrag des Aristoteles nicht in seiner urkundlichen Fassung direkt gekannt hat, beweist die falsche Zahlenangabe der Mitglieder der Kommission der Dreissig.

3) Im Sinne der Identität der beiden Anträge besonders Eduard Meyer und Köhler. Schärfer wird von Busolt, Griechische Geschichte III, 21479 ff. Anm. die Inhaltsverschiedenheit des aristotelischen und thukydideischen' Antrags hervorgehoben, ja wenigstens logisch der Antrag des Peisander von den der Dreissig völlig abgetrennt. Ich halte darum die Darstellung Busolts am meisten der Wahrheit nahekommend. Nur handelt es sich bei Busolt, besonders S. 1482 Text, wie auch bei Ed. Meyer, Forschungen II, 429 f. immer nur um Vermutungen und Hypothesen. Durch die oben versuchte Interpretation des Antrags Peisanders, dessen zeitlicher und realer Verschiedenheit von dem bei Aristoteles vorliegenden Antrag der Dreissig würden diese Vermutungen, wie mir scheint, als volle geschichtliche Wahrheit bewiesen.

4) Forschungen II, 419. - 5) Griechische Geschichte III, 2 S. 1479 Anm. 2.

6) Sitzungsberichte 1900 S. 808.

K 1 i o, Beiträge zur alten Geschichte VII 3. 
sondern in dem, dass der bei Thukydides überlieferte Antrag des Peisander überhaupt erst zeitlich später dem bei Aristoteles erhaltenen materiellen Antrag der Kommission der Dreissig als ergänzender, umgestaltender $\mathrm{Zu}-$ satzantrag hinzugefügt ist. Dass daher Thukydides irrt, wenn er die Kommission der Dreissig nur betreffs des vorbereitenden Antrags auf Auf-

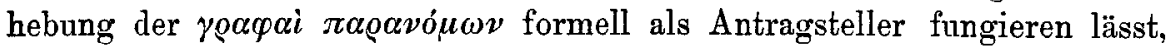
ist nach den bei Aristoteles vorliegenden Mitteilungen nicht zu bestreiten. Daraus wird zugleich ferner ersichtlich, dass Peisander unmöglich, wie noch Eduard Meyer und Busolt ${ }^{1}$ ) annebmen, der offizielle Redner der Kommission der Dreissig gewesen sein kann. Dazu standen ja auch die von Peisander gemachten Vorschläge allzusebr der Tendenz der von Aristoteles ïberlieferten Kommissionsbestimmungen entgegen.

Aus Thukydides' Bericht ergibt sich ferner, dass in Peisanders Antrag neben der offenbar aus dem materiellen Antrag der Dreissig übernommenen Forderung der Abschaffung der Besoldung überdies eine völlige Reorganisation der Aemter vorgesehen war. Auch hierin hat Köhler, da die betreffende Angabe bei Aristoteles fehlt, nur einen Irrtum des Thukydides erblicken wollen. In dem bei Aristoteles überlieferten Verfassungsentwurf für die Zukunft ist ,eine Umgestaltung der höchsten Beamtenkollegien, insbesondere der Finanz- und Polizeibehörden vorgeschrieben. Unter diesen Umständen muss man urteilen, dass in dem Bericht des Thukydides Frïheres mit Späterem vermengt “ ist ${ }^{2}$ ). Indes kann ja bei der völligen Verschiedenheit des thukydideischen und aristotelischen Antrags dieser unmöglich als Kriterium des von Peisander gestellten Antrags verwertet werden, und umgekehrt erhellt aus dem bei Aristoteles mitgeteilten Verfassungsentwurf für die 5000, dass die daselbst formulierte Reorganisation der Aemter formell bereits in dem Antrag Peisanders aufgenommen war. Von dort ist sie erst als leitende Bestimmung in den Verfassungsentwurf für das Definitivum der 5000 eingedrungen.

In Widerspruch zu Aristoteles wie der Rede für Polystratos steht ferner die nach Thukydides Bericht durch die fünf Proedroi zu erfolgende Wahl der 100. Denn dass die Hundert tatsächlich von den Phylen, nicht von den Proedroi gewählt wurden, ist nach den ausdrücklichen Angaben der Rede für Polystratos nicht zweifelhaft. Dementsprechend ist auch die von Thukydides gemachte Mitteilung über die Proedroi umzuändern. Ed. Meyer ${ }^{3}$ ) vermutet, dass die Proedroi in Wirklichkeit nicht die aktiven Wähler, sondern nur die Leiter der tatsächlich durch die Phylen vollzogenen Wahl der Kommission von 100 gewesen sind, mögen sie faktisch einen auch noch so grossen Einfluss auf die Wahl selbst im Sinne der oligarchischen Verschwörung ausgeübt haben. Indessen ist bereits

1) Griechische Geschichte III, 2 S. 1479.

2) Sitzungsberichte 1900 S. 809.

3) Forschungen II, $430 \mathrm{f}$. 
von Köhler ${ }^{1}$ ), dann von Busolt ${ }^{2}$ ) mit Recht dieser Rettungsversuch Meyers aus sprachlichen Gründen - zweifellos bedeutet das $\tau o v i \tau o v \varsigma ~ \delta \dot{\varepsilon} \varepsilon \dot{\varepsilon} \dot{\varepsilon} \sigma \vartheta \alpha \iota$

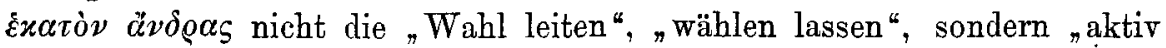
wählen" - zurückgewiesen worden. Weit mehr Zustimmung hat daher die Vermutung $G$. Gilberts ${ }^{3}$ ) gefunden, der zufolge sich in den fünf Proedroi der leitende Ausschuss der Oligarchen darstellt - eine Oberleitung, die ja zweifellos bei dem Erfolge, den die Oligarchen gehabt haben, tatsächlich bestanden haben muss. Allein auch dieser Erklärung stellt sich die Schwierigkeit entgegen, dass bei Thukydides die Stellung und Aufgabe der Proedroi eine staatsrechtlich und offiziell festumschriebene ist, während, wie Wilamowitz ${ }^{4}$ ) sehr richtig hervorhebt, die fünf Proedroi im Sinne des leitenden Ausschusses der Klubbisten rechtlich und offiziell unmöglich bestehen konnten. Es ist besonders nach den Angaben der Rede für Polystratos kein Zweifel, dass in diesen Bestimmungen des Antrags bei Thukydides ein geschichtlich zweifellos nicht verwirklichtes Moment vorliegt. Mögen wir uns dieselben nach den obigen Ausführungen als ein rudimentäres Glied des ursprünglich schriftlichen und dann offiziell abgeänderten Antrags des Peisander oder sonst wie erklären, es genügt für die Feststellung des historischen Vorgangs zu bemerken, dass die Kommission der 100 tatsächlich von den Phylen gewählt worden ist, und nur die in dem Verfassungsentwurf für die 5000 bei Aristoteles cap. 30 erhaltene Bestimmung der Leitung der Ratsverhandlungen durch einen Ausschuss von fünf Münnern lässt uns jene Mitteilung bei Thukydides auch in einem verfassungsgeschichtlich interessanteren Lichte erscheinen. Demnach ist etwas Aehnliches wie in dem Verfassungsentwurf für die 5000 schon in dem Antrag des Peisander beabsichtigt gewesen. Und wie ferner Ed. Meyer aus dem Ehrendekret des Pythophanes von Karystos (CIA II, 1c p. 396) erkannt hat, haben fünf Proedroi als Leiter des Rats auch tatsächlich während der kurzen Herrschaft der 9000 nach dem Sturz der 400 bestanden ${ }^{5}$ ).

Alles weitere ist schon von Ed. Meyer mit völliger Sicherheit erörtert worden. Vor allem erhellt, dass betreffs der faktischen Existenz der 400 und der bloss nominellen Auswahl der 5000 während des Regimentes der 400 allein Thukydides recht unterrichtet ist. Was uns Thukydides erzählt, dass zunächst die Kommission von 100 durch Kooptation sich zu einem Rat von 400 konstituiert und dieser mit unumschränkter Machtvollkommenheit die Staatsgewalt ausübt, wird vollkommen durch Polystratos' Verhalten bestätigt. Auch dass, wie wir aus Thukydides schliessen müssen, in dem Rat der 400 zwei Kategorien vorhanden sind: 100 von den Phylen Gewählte und 300 von den Hundert Ernannte, wird, sobald

1) Sitzungsberichte 1900 S. 811 Anm. - 2) Griechische Geschichte III, 2 S. 1480 Anm.

3) Beiträge zur inneren Geschichte Athens S. 307.

4) Aristoteles und Athen II, 358. - 5) Forschungen II, $430 \mathrm{f}$. 
wir nur mit völliger Berechtigung die Zahl 100 von der aristotelischen

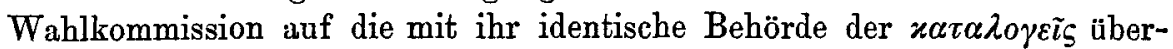
tragen, durch die Rede für Polystratos bewiesen. Alles, was dagegen Aristoteles bietet, stellt sich als subjektive, falsche Schlussfolgerung aus den Akten dar. Vor allem ergibt sich, dass die Konstituierung der Wahlkommission von 100 und die durch diese erfolgte Einsetzung des Rates der 5000, die der Eingang cap. 30 zwar nicht selbst schildert, wohl aber voraussetzt, keineswegs in den Akten selbst als Tatsache enthalten, sondern lediglich aus ihnen von Aristoteles oder Aristoteles' Quelle gefolgert ist. Die in cap. 30, 31 vorliegenden Verfassungsentwürfe für die provisorische Regierung der 400 und den definitiven Rat der 5000 geben sich ferner als Vorlage einer von den 5000 eingesetzten Kommission von Hundert. So folgert Aristoteles, dass erst tatsächlich die 100 von den $\mathbf{5 0 0 0}$ eingesetzt, dann gemäss den Verfassungsentwürfen das Regiment der 400, endlich nach diesen die Herrschaft der 5000 geschichtlich realisiert worden ist, und nur die weitere Angabe, dass die 5000 doch nur dem Namen nach ernannt worden sind, lässt schliesslich den aristotelischen Bericht in dem Lichte völliger Brüchigkeit erscheinen. Nachdem der in Aussicht genommene Rat der 5000 die Verfassungskommission der Hundert eingesetzt, also tatsächlich konstituiert sein muss, endet der aristotelische Bericht in dem sich selber vernichtenden Geständnis: oi $\mu \dot{\varepsilon} \nu \pi \varepsilon \nu \tau a \varkappa \iota \sigma \chi i \lambda \iota \iota \iota$

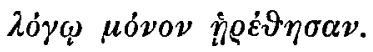

Uebrigens wird uns die bloss nominelle Existenz der 5000 auch sonst durch die Rede für Polystratos bewiesen. Zwar sucht die Verteidigung für die Sache des Angeklagten dadurch zu gewinnen, dass nach ihrer Darlegung Polystratos an einer Auswahl von 9000 teilnimmt (13), und eine

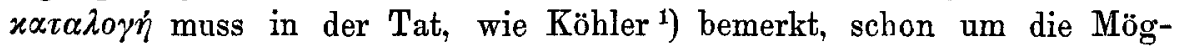
lichkeit dieser Angabe zu erklären, stattgefunden haben. Nur ist das nicht eine $\varkappa \alpha \tau \alpha \lambda o \gamma \eta^{\prime}$ unter dem Regiment der 400, sondern die Auswahl der 5000 resp. $9000^{2}$ ) nach dem Sturz der Vierhundert. Dass Polystratos an dieser $\varkappa \alpha \tau \alpha \lambda \circ \gamma \eta$ nicht beteiligt gewesen ist, beweisen die eigenen $\mathrm{An}$ -

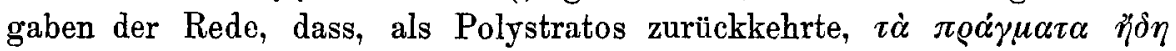

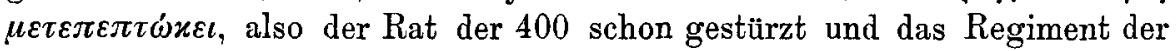
gemässigt oligarchischen Verfassung bereits eingerichtet ist (14). Als pures Blendwerk ist die $\varkappa \alpha \tau \alpha \lambda o \gamma \dot{\eta}$ der 9000 von der Verteidigung an die falsche Stelle gesetzt worden.

Nur kurz möge noch auf das von Aristoteles namhaft gemachte Interregnum vom 14.-22. Thargelion eingegangen werden. Wie Ed. Meyer ${ }^{3}$ ) sehr richtig erkannt hat, bezieht sich die von Aristoteles im Eingang cap. 32 genannte Vollversammlung unter dem Vorsitz des Aristomachos

1) Sitzungsberichte 1900 S. 814.

2) Beloch, Bevölkerung der griechisch-römischen Welt S. $107 \mathrm{f}$.

3) Forschungen II, 432. 
zweifellos auf die Volksversammlung auf dem Kolonos. Nur so erklärt es sich, dass sich an diese Versammlung genau so wie bei Thukydides auch bei Aristoteles die Auflösung des alten Rates unmittelbar anschliesst ${ }^{1}$ ). Bedenken möchte dagegen das nach Aristoteles eingetretene Interregnum vom 14.-22. Thargelion erregen. Dass in der Tat dies Interregnum, wie Aristoteles die Dinge darstellt, ohne vorherige faktische Konstituierung der 400 realiter ein Ding der Unmöglichkeit ist, ist schon oben bemerkt worden. Indessen findet sich, wenn wir die Auflösung des alten Rates bei Aristoteles am 14. Thargelion im Sinne des thukydideischen Berichtes auffassen, bei Thukydides etwas Aehnliches angedeutet. Auf eine Zeit, wo die Verschworenen faktisch in den Besitz der Gewalt und des Rathauses gelangt sich lediglich der Erledigung formeller Angelegenheiten, der Wahl der Prytanen, der Darbietung von Gebeten und Opfern unterziehen, folgt das eigentliche Schreckensregiment der 400, die Ermordung und Verbannung der Missliebigen, der Einkerkerung der Gegner und sonstiger Aenderungen in den Verordnungen (cap. 67,3). Genau dasselbe wird uns jetzt durch die Rede für Polystratos bestätigt. Wir erfabren aus dieser, dass Polystratos sofort nach abgelegtem Amtseid in den Rat eintritt, allein nur 8 Tage an den Sitzungen des Rates - nach der Darstellung der Verteidigung sogar nur schweigend - teilnimmt, dann als Kommandant nach Eretria abfährt. Diese 8 Tage der Ratsanwesenheit des Polystratos sind ohne Zweifel mit dem von Aristoteles namhaft gemachten und auch aus Thukydides indirekt ersichtlichen Interregnum vom 14.-22. Thargelion identisch. Offenbar hat man sich in dieser Zwischenzeit damit beschäftigt, die formellen Grundlagen des neuen Regiments zu schaffen, die nötigen Beamten zu wählen und die Besetzung der wichtigsten Exekutivposten mit absolut zuverlässigen Gesinnungsgenossen vorzunehmen. So hat Polystratos - offenbar als zuverlässiger Oligarch - das wichtige Amt des Kommandanten von Eretria erhalten. In diese Zeit muss ferner die Ausarbeitung der bei Aristoteles überlieferten Verfassungsentwürfe für das provisorische Regiment der 400 und das definitive Regiment der 5000 fallen. Denn als man nach Thukydides' Bericht in möglichster Beschleuni-

1) Wäre letzteres nicht, so würde man geneigt sein, mit Volquardsen a. a. 0. S. 128 ff. die bei Aristoteles im Anfang cap. 32 erwähnte Bestätigung allerdings auf die in cap. 30, 31 enthaltenen Verfassungsentwürfe zu beziehen und ihre Annahme in die Zeit zwischen den 14. und 22. Thargelion zu setzen. Um dies durchzuführen, muss Volquardsen annehmen, dass von Aristoteles die Vorgänge zwischen dem 14. und 22. Thargelion irrtümlicherweise vor den erstgenannten Tag gesetzt worden sind. Dem steht gegenüber, dass bei dieser Auslegung unter $\pi \lambda \pi \vartheta 0 \varsigma$ nur die Vollversammlung des Rates der Vierhundert, nicht aber der alten abgedankten Volksversammlung noch der, wie wir aus der Rede für Polystratos mit Sicherheit wissen, überhaupt nicht existierenden Fünftausend verstanden werden kann (gegen Volquardsen a. a. O. S. 125 und 128). Gerade diese Auslegung ist aber nach den gesamten Angaben der aristotelischen Ueberlieferung, ihrer sonstigen geschichtlichen Wahrscheinlichkeit ungeachtet, vollkommen unmöglich. 


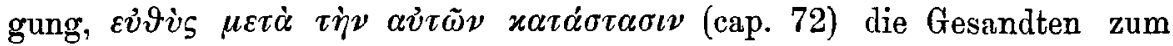
Heer nach Samos zur Beschwichtigung abschickt, werden diesen die Instruktionen bereits im Sinne der in cap. 30 enthaltenen Verfassung für die 5000, der sektionsweise sich ablösenden Regierung der vier Ratsabteilungen nitgegeben. Demgemäss reden die Gesandten zu dem Heere

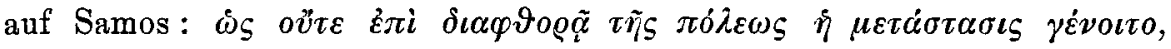

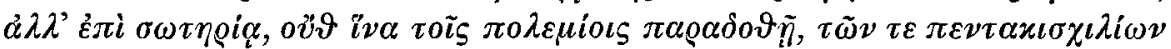

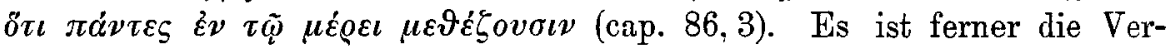
mutung Ed. Meyers ${ }^{1}$ ), dass nach diesen vorbereitenden Aktionen der Rat der 400 rechtlich erst am 22. Thargelion, entsprechend der Datumsangabe des aristotelischen Berichtes, die Regierung ergriffen und erst an diesem Tage sich offiziell konstituiert hat. $\mathrm{Ob}$ dabei die 400 noch einmal gemäss der Bestimmung des aristotelischen Verfassungsentwurfes cap. $31 \dot{\varepsilon} x \pi \varrho 0-$ $\varkappa$ ¿i $\omega \nu$ von den Phylen gewählt wurden, mag dahingestellt bleiben. Sicher ist nur, dass das Regiment der 400 offiziell erst vom 22. Thargelion datiert. Und damit beginnt, wie wir aus Thukgdides ersehen, zugleich die Zeit eines gewalttätigeren Vorgehens der Vierhundert.

Die Rede für Polystratos ist zugleich der unumstössliche Beweis dafür, dass nicht nur in dem Tatsachenbestand, sondern auch dem Kolorit, der stimmungsreichen Wiedergabe der Geschehnisse allein Thukydides das Richtige getroffen hat. Der Schrecken der Revolution, die Gewalttätigkeit der Machthaber, die Furcht und Angst der Nichtverschworenen, Mord und Einkerkerung, die in dem offiziellen Aktenbericht des Aristoteles keine Darstellung, nicht einmal in seiner Schilderung eine Erwähnung finden, sie hallen uns aus dem Bericht des Thukydides genau so wie aus der Rede für Polystratos offenkundig entgegen. Polystratos ist Mitglied des Rats, aber, wie die Verteidigung angibt, nur gezwungen. Anders zu handeln, war ihm bei dem Terrorismus der Machthaber unmöglich, denn wer sich

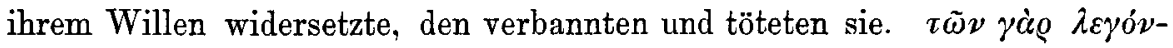

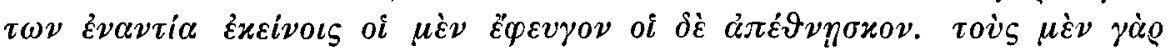

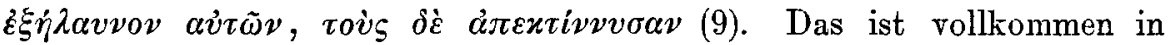
Uebereinstimmung mit dem Kolorit des thukydideischen Berichtes - ein Beweis wieder für die glänzende Skizzierung, in der der gewaltige Meister antiker Geschichtsschreibung nicht nur die treibenden Motive und grundlegenden Begebenheiten unbeirrt durch die reale Inkongruenz der Akten, sondern auch die gesamte Stimmung, das reiche Bild des buntbewegten Treibens in seinen mannigfaltigen geheimnisvollen wie offiziellen Zügen dargestellt hat ${ }^{2}$ ).

1) Forschungen II, 425.

2) Ein Beweisgrund der neuerdings öfters behaupteten formellen wie inhaltlichen Mangelhaftigkeit des achten Buches bei Thukydides wird man also aus Thukydides' Darstellung der Revolution der Vierhundert schwerlich herleiten können. Eine nähere Erörterung des eben berührten Problemes liegt im übrigen ausserhalb des Rahmens dieser in bewusster Absicht unabhängig von jener Frage angestellten Untersuchungen. 